\title{
Quasistatic Crack Propagation in Heterogeneous Media
}

\section{Citation}

Ramanathan, Sharad, Deniz Ertaș, and Daniel S. Fisher. 1997. “Quasistatic Crack Propagation in Heterogeneous Media." Physical Review Letters 79 (5): 873-76. https://doi.org/10.1103/ physrevlett.79.873.

\section{Permanent link}

http://nrs.harvard.edu/urn-3:HUL.InstRepos:41467461

\section{Terms of Use}

This article was downloaded from Harvard University's DASH repository, and is made available under the terms and conditions applicable to Other Posted Material, as set forth at http:// nrs.harvard.edu/urn-3:HUL.InstRepos:dash.current.terms-of-use\#LAA

\section{Share Your Story}

The Harvard community has made this article openly available.

Please share how this access benefits you. Submit a story.

Accessibility 


\title{
Quasi-static crack propagation in heterogeneous media
}

\author{
Sharad Ramanathan, Deniz Ertaş and Daniel S. Fisher \\ Lyman Laboratory of Physics, Harvard University, Cambridge, Massachusetts 02138
}

(November 20, 1996)

\begin{abstract}
The dynamics of a single crack moving through a heterogeneous medium is studied in the quasistatic approximation. Equations of motion for the crack front are formulated and the resulting scaling behaviour analyzed. In a model scalar system and for mode III (tearing) cracks, the crack surface is found to be self affine with a roughness exponent of $\zeta=1 / 2$. But in the usual experimental case of mode I (tensile) cracks, local mode preference causes the crack surface to be only logarithmically rough, quite unlike those seen in experiments. The effects of residual stresses are considered and found, potentially, to lead to increased crack surface roughness. But it appears likely that elastic wave propagation effects may be needed to explain the very rough crack surfaces observed experimentally.
\end{abstract}

PACS numbers:62.20.Mk, 03.40.Dz, 46.30.Nz, 81.40.Np

Since the work of Mandelbrot, Passoja and Paullay [1], many experimental [2 [4] and numerical [5] studies on geometrical properties of crack surfaces and crack fronts in heterogeneous materials have demonstrated their selfaffine scaling properties. Cracks, in a large variety of materials from aluminum alloys [2] to ceramics [3] and rock [4], seem to leave behind surfaces whose height variations are characterized by a roughness exponent $\zeta \approx 0.8$. In the meantime, there have been significant advances in the understanding of the dynamics of interfaces and lines pinned by quenched random impurities [6] 8 . Potentially, these might shed light on the problem of crack surfaces and fronts [9], since a crack surface can be viewed as the trace left behind by the crack front as it traverses the sample. But such theoretical analyses of crack surfaces have so far neglected the crucial long range effects of elasticity. In particular, the deformations of a crack front change stresses at distant points on the front [10]. Moreover, the stresses are also modified by the boundary conditions on the rough crack surface, which give rise to dependence on the history of the crack front. As the crack moves, it relieves both the elastic stresses generated by the external loading and those due to the presence of quenched impurities such as inclusions, micro-cracks or dislocations. Because of elasticity, these defects give rise to long-range correlated randomness in the equations of motion.

All of these effects influence the macroscopic scaling behavior of the crack surface and have to be included in a realistic model of crack propagation. Unfortunately, the full elastodynamic problem is very difficult. Indeed, even the criteria that determine the local direction of advance of crack front are not well understood [11].

In this paper, we present the results of a study of a crack propagating through a heterogeneous medium in the quasi-static approximation. We first consider a simplified scalar elasticity theory and then the real case of vectorial elasticity. We use the symmetries of the system and elasticity theory to determine phenomenological equations of motion.

Our conclusions are, unfortunately, that for the principal situation of experimental interest of tensile (mode I) cracks [and also shear (mode II) cracks] the predicted roughness of the crack front is only logarithmic rather than a power law of the length scale. Tearing (mode III) cracks are substantially rougher; however, these tend not to be stable experimentally and thus the applicability of the results is questionable. In all cases, random long wavelength residual stresses can increase the roughness; the effects of these are discussed briefly.

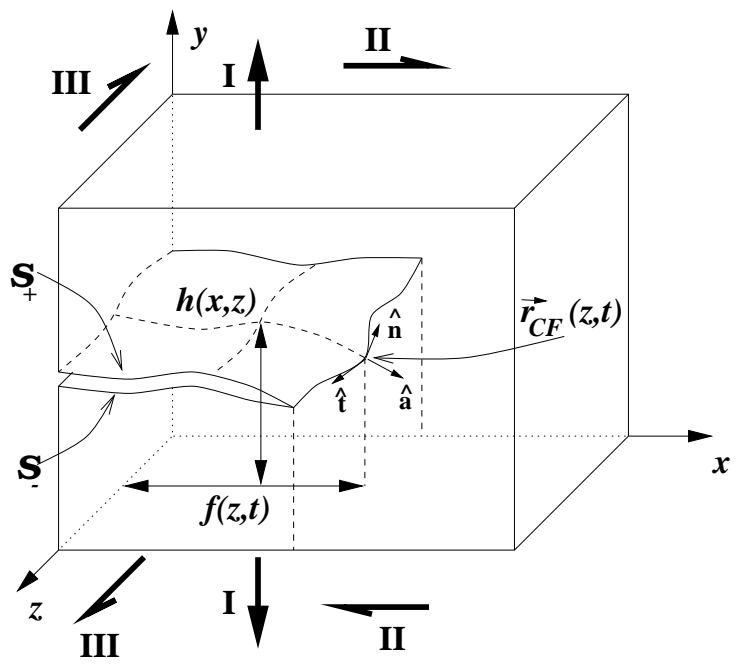

FIG. 1. Crack propagating through a heterogeneous medium. The $x$ co-ordinate of the front is $f(z, t)$ and the free crack surfaces $\mathcal{S}_{+}, \mathcal{S}_{-}$are located at $y=h(x, z)$. The local tangent, forward and normal vectors $\hat{\mathbf{t}}, \hat{\mathbf{a}}, \hat{\mathbf{n}}$ at a point $\mathbf{r}_{C F}$ on the front are also shown as are the directions of mode I (tensile), mode II (shear) and mode III (tearing) loading.

The geometry of the crack is shown in Fig. 1. The crack front is oriented along the $z$-axis and moves in the positive $x$ direction. The $x$ (in-plane) coordinate of the front at time $t, x_{c}(z, t)=f(z, t)$ is assumed to be single valued in $z$. As the crack moves, it leaves behind free surfaces $\mathcal{S}_{+}$and $\mathcal{S}_{-}$on either side of the crack, located in body coordinates at $y=h(x, z)$ for $x<f(z, t)$. Thus, the instantaneous position of the front is given by the curve $\mathbf{r}_{C F}(z, t) \equiv f(z, t) \hat{\mathbf{x}}+h[f(z, t), z] \hat{\mathbf{y}}+z \hat{\mathbf{z}}$. We assume that the crack motion is quasi-static, so that at each instant, the scalar displacement field $u(x, y, z)$ satisfies Laplace's equation with the boundary condition that the normal stresses on the surfaces $\mathcal{S}_{+}$and $\mathcal{S}_{-}$vanish. The sample 
is loaded far away from the crack, such that

$$
u \approx \frac{K^{\infty} y}{2 M \sqrt{\pi}} \sqrt{\frac{\sqrt{x^{2}+y^{2}}-x}{y^{2}}},
$$

for $\sqrt{x^{2}+y^{2}}$ large, with $K^{\infty}$ the applied stress intensity factor and $M$ the elastic modulus. Displacement and stress fields in the medium are determined by the position of the front and the shape of the crack surface and these in turn influence the subsequent motion of the crack. We now derive general equations of motion for the time evolution of the crack and analyze them to determine scaling exponents that characterize the roughness of crack fronts and surfaces.

A local coordinate system can be defined at a point $\mathbf{r}_{C F}$ on the crack front, with axes along the tangent $\hat{\mathbf{t}} \equiv$ $\partial_{z} \mathbf{r}_{C F} /\left|\partial_{z} \mathbf{r}_{C F}\right|$, forward direction $\hat{\mathbf{a}} \equiv \partial_{z}^{2} \mathbf{r}_{C F} /\left|\partial_{z}^{2} \mathbf{r}_{C F}\right|$, and normal $\hat{\mathbf{n}} \equiv \hat{\mathbf{t}} \times \hat{\mathbf{a}}$ (see Fig. 1). The direction in which the crack front moves should be governed by the angular dependence of the stress and the local yield stress near the crack tip. The stress field close to the crack tip has the form

$$
\boldsymbol{\sigma}=\frac{K\left(\mathbf{r}_{C F}\right)}{\sqrt{2 \pi \rho}}[\cos (\theta / 2) \hat{\mathbf{n}}-\sin (\theta / 2) \hat{\mathbf{a}}]+S_{a}\left(\mathbf{r}_{C F}\right) \hat{\mathbf{a}}+\mathcal{O}(\sqrt{\rho}),
$$

with $\rho$ the distance to the crack front and $\theta$ the angle with respect to $\hat{\mathbf{a}}$ in the $(\hat{\mathbf{a}}-\hat{\mathbf{n}})$ plane. The local stress intensity factor is $K\left(\mathbf{r}_{C F}\right)$ and $\mathbf{S}$ is the finite part of the stress whose normal component $S_{n}$ must vanish. The divergent part of the stress field does not break the reflection symmetry with respect to the crack front plane (spanned by $\hat{\mathbf{t}}$ and $\hat{\mathbf{a}}$ ), so the crack should move in the forward direction â leaving behind a crack surface that is smooth on the smallest length scales.

As the crack front advances, an elastic energy $G\left(K^{\infty}, \mathbf{r}_{C F},\{f\},\{h\}\right)=\frac{K^{2}\left(\mathbf{r}_{C F}\right)}{M}$ per unit area of generated crack surface is released by the medium. In order to create the new crack surface, a surface energy $\Gamma\left(\mathbf{r}_{C F}\right)$ per unit area is required. This is a local material property. We will assume that the zone with non-linear deformations, plasticity or microscopic side branches is small and that linear elasticity theory is applicable outside of this region; $\Gamma$ will include the energy to break bonds, as well as that dissipated as a result of plastic flow etc. Under quasi-static conditions, the forward velocity $v_{a} \equiv \hat{\mathbf{a}} \cdot \partial_{t} \mathbf{r}_{C F}$ of the crack front is proportional to the excess elastic energy released, i. e.,

$$
\mu^{-1} v_{a}\left(\mathbf{r}_{C F}\right)=G\left(K^{\infty}, \mathbf{r}_{C F},\{h\},\{f\}\right)-\Gamma\left(\mathbf{r}_{C F}\right),
$$

where $\mu$ is the effective mobility of the crack front. If $\mu$ is much smaller than $(c \Gamma)^{-1}$, with $c$ the speed of sound, the quasistatic approximation should be good except on very long length scales.

Energy considerations alone cannot determine how the direction of motion of the crack front changes. Instead, we expect this to be determined by the small scale processes that break the reflection symmetry about the local crack surface. In particular, the finite part of the stress field near the crack front, $S_{a}\left(\mathbf{r}_{C F}\right)$ in Eq.(2) is antisymmetric about the crack surface. The competition of this with the local random variations in the material yield stress will determine the curvature $\kappa \equiv \hat{\mathbf{n}} \cdot \partial_{a} \hat{\mathbf{a}}$ of the path of the crack front as it advances: $S_{a}$ will tend to make the crack curve in the direction in which the "hoop" stress $\sigma_{\theta}$ or the total stress $|\sigma|$ is larger. For small changes in the direction of advance, this will be determined by the stresses near $\theta=0$; from the small $\theta$ behaviour of Eq.(2), we thus see that $\kappa$ should be proportional to $-S_{a}$. But the yield stress ( or other appropriate fracture stress) of the material, $\sigma_{\mathrm{Y}}$, can vary with position and the crack will tend to bend in a direction in which $\sigma_{\mathrm{Y}}$ is lower. We postulate that the competition between these effects determines the local curvature:

$$
\kappa=-\left(S_{a}+b \partial_{n} \sigma_{\mathrm{Y}}\right) / \lambda,
$$

with $b \sim K_{c}^{2} / \sigma_{\mathrm{Y}}^{2} M^{2}$ of order the distance from the crack tip at which the stress is $\sigma_{\mathrm{Y}}$ and $\lambda \sim K_{c} \sqrt{b}$, with $K_{c}$ the critical stress intensity factor for crack advance. The detailed form of Eq.(A) will turn out to be unimportant at long length scales.

Both $S_{a}$ and the energy release rate, $G$, can be written as a sum of two terms. The first terms, $G^{u}$ and $S_{a}^{u}$ are the energy release rate and the nonsingular stress near the crack front for a homogeneous medium with the same loading and geometry of the crack surface, and thus do not depend on the heterogeneities in the system. These are functionals of the entire shape of the front and the crack surface, as well as the applied load. The second terms, $G^{r}$ and $S_{a}^{r}$ are the contributions from the relief of frozen-in stresses due to the heterogeneities and defects in the medium as well as any elastic inhomogeneities.

In general, $G^{r}$ and $S_{a}^{r}$ will also depend on the crack shape. But for almost straight cracks, they will primarily depend on the unperturbed shape of the crack and to linear order in the randomness this effect can be neglected. We can now define random variables

$$
\begin{aligned}
\chi(x, z) & \equiv b \partial_{n} \sigma_{\mathrm{Y}}[x, h(x, z), z]+S_{a}^{r}[x, h(x, z), z], \\
\gamma(x, z) & \equiv \Gamma[x, h(x, z), z]-\bar{\Gamma}-G^{r}[x, h(x, z), z],
\end{aligned}
$$

on the crack surface, with $\left\langle G^{r}\right\rangle=\left\langle S_{a}^{r}\right\rangle=0$ and $\langle\Gamma\rangle=\bar{\Gamma}$, the mean fracture toughness. These will depend on $h(x, z)$ but to lowest order in $h$ this effect is not important. The means of $\gamma$ and $\chi$ are defined to be zero and their correlations are taken to be

$$
\begin{aligned}
& \left\langle\gamma(x, z) \gamma\left(x^{\prime}, z^{\prime}\right)\right\rangle=\Delta\left(x-x^{\prime}, z-z^{\prime}\right) \\
& \left\langle\chi(x, z) \chi\left(x^{\prime}, z^{\prime}\right)\right\rangle=\Upsilon\left(x-x^{\prime}, z-z^{\prime}\right) .
\end{aligned}
$$

We first consider short range correlations where $\Delta$ and $\Upsilon \propto \delta\left(x-x^{\prime}\right) \delta\left(z-z^{\prime}\right)$,with some short distance cut-off, later returning to the effects of long range correlations. The loads and the shape of the crack surface determine the stress field. Consequently Eqs.(3-8) determine the evolution of the crack front. 
We have obtained a solution for the displacement field perturbatively around a flat, straight crack in an isotropic, homogeneous medium that can be carried out order by order in $h(x, z)$ and $f(z, t)$ [12]. This solution combined with Eq.(3) yields an equation of motion for $f(z, t)$. To linear order, out-of-plane deviations of the crack surface do not contribute to the divergent stress just ahead of the crack tip that determines the energy release rate. The behavior of the in-plane displacement $f$ is identical to that of a crack which is restricted to move in a plane:

$$
\begin{aligned}
\mu \partial_{t} f(z, t)= & \frac{\sqrt{G^{\infty}}}{\pi} \mathcal{P} \int_{-\infty}^{\infty} d z^{\prime} \frac{f\left(z^{\prime}, t\right)-f(z, t)}{\left|z-z^{\prime}\right|^{2}} \\
& -\gamma(f(z, t), z)+G^{\infty}-\bar{\Gamma}
\end{aligned}
$$

with $G^{\infty}=\left(K^{\infty}\right)^{2} / M$ and $\mathcal{P}$ denotes the principal part of the integral. Similarly, Eq.(化) gives us an equation for the evolution of $h(x, z)$. Stresses generated by in-plane fluctuations of the crack front do not break the reflection symmetry and therefore cannot affect the curvature $\kappa$. Thus, the leading correction to $S_{a}^{u}$ involves only out-ofplane fluctuations $h$. The linearized equation for the evolution of $h$ as the front passes a point $\mathbf{r}_{C F}$ at $x=f(z, t)$ is

$$
\begin{aligned}
\lambda \partial_{x}^{2} h(x, z)= & \sqrt{G^{\infty}} \mathcal{P} \int_{-\infty}^{\infty} d x^{\prime} \int_{-\infty}^{\infty} d z^{\prime} J\left(x-x^{\prime}, z-z^{\prime}\right) h\left(x^{\prime}, z\right) \\
& -\chi(x, z)
\end{aligned}
$$

independent of $f$. The Fourier transform of the long range kernel $J$ is

$$
\tilde{J}(q, k)=(q-i \epsilon)^{3 / 2} Y(k / q),
$$

with $Y$ a scaling function. To linear order the equations of motion for $f$ and $h$ decouple. Therefore the crack surface is determined independently of the dynamics of the front, which is not true in general.

Equation (9) has been studied both analytically [8] and numerically [12]. The front is arrested for small external loads, and there is a critical load $K_{c}^{\infty}$ (corresponding to $G_{c}^{\infty}$ ) at which the crack just begins to move. For a load $K^{\infty}$ slightly above this threshold, the average velocity of the front scales as $v \sim\left(K^{\infty}-K_{c}^{\infty}\right)^{\beta}$. The motion of the front is rather jerky with fluctuations in the velocity correlated up to a distance $\xi$, which diverges at threshold like $\xi \sim\left(K^{\infty}-K_{c}^{\infty}\right)^{-\nu}$. At length scales smaller than $\xi$, the in-plane front profile $f(z, t)$ is selfaffine with $\left\langle\left[f(z, t)-f\left(z^{\prime}, t\right)\right]^{2}\right\rangle \sim\left|z-z^{\prime}\right|^{2 \zeta_{f}}$. A renormalization group $\epsilon$-expansion and numerical simulations suggest $\zeta_{f}=1 / 3, \nu=3 / 2, \beta \approx 7 / 9$. At length scales larger than $\xi$ (or well above threshold), the crack front moves more uniformly, and its in-plane fluctuations scale as

$$
\left\langle\left[f(z, t)-f\left(z^{\prime}, t\right)\right]^{2}\right\rangle \sim \log \left|z-z^{\prime}\right|,
$$

as can be seen by Fourier transforming Eq.(9).

In order to calculate scaling properties of the crack surface, we next analyze Eq.(10). At length scales larger than $b$, the left hand side of Eq.(10) becomes negligible and the direction in which the crack moves is determined by the competition between the non singular stress and the material properties near the crack front. Thus, beyond this length scale our results will be the same if we alternatively assume that the crack tip moves in a direction in which the stress $\sigma_{\theta}$ exceeds the local material yield stress furthest from the crack front. The crack surface roughness thus has the scaling form

$$
\left\langle\left[h(x, z)-h\left(x^{\prime}, z^{\prime}\right)\right]^{2}\right\rangle \sim\left|z-z^{\prime}\right|^{2 \zeta_{h}} H\left(\left|x-x^{\prime}\right| /\left|z-z^{\prime}\right|\right),
$$

with $H$ a scaling function. The crack surface is anisotropic but with the same roughness exponent of $\zeta_{h}=0.5$ in both the $x$ and the $z$ directions.

We now consider the real case of vectorial elasticity. Near the crack front, the stresses have the form

$$
\sigma_{i j}=\sqrt{\frac{1}{2 \pi \rho}}\left\{K_{\mathrm{I}} \Sigma_{i j}^{\mathrm{I}}(\theta)+K_{\mathrm{II}} \Sigma_{i j}^{\mathrm{II}}(\theta)+K_{\mathrm{III}} \Sigma_{i j}^{\mathrm{III}}(\theta)\right\},
$$

where the stress intensity factors $K_{\mathrm{I}}, K_{\mathrm{II}}$ and $K_{\mathrm{III}}$ are associated, respectively, with discontinuities across the crack surface of displacements in the $\hat{\mathbf{n}}$ (tensile loading), $\hat{\mathbf{a}}$ (shear loading) and $\hat{\mathbf{t}}$ (tear loading) directions; and the $\Sigma_{i j}^{\mu}$ are universal functions. If the crack is loaded far away purely in mode III, then out of plane $z$-independent deformations $h(x)$ in the crack surface will not mix in the other modes near the crack front. The direction in which the crack progresses will then, as for the scalar case, be determined by the finite non-singular parts of the stress near the tip. The roughness exponents should thus be the same as the scalar case although the scaling functions will be different. Unfortunately, mode III cracks tend to be unstable [13], so the applicability of these results is questionable.

The primary situation of experimental interest is mode I (tensile) loading. In this case, once the crack wanders out of plane, the local $K_{\mathrm{II}}$ becomes non-zero, and with $z$-dependent distortions of the crack, so does $K_{\mathrm{III}}$. To linear order, $K_{\text {II }}$ and $K_{\text {III }}$ are functionals of $h$ while $K_{\text {I }}$ only depends on $f$. As the crack moves, the change in the energy release rate $G=\sum_{\mu} \frac{K_{\mu}^{2}}{M_{\mu}}$, where $M_{\mu}$ are the appropriate elastic constants [14], is dominated by $\delta K_{\mathrm{I}}$. Therefore, as in the scalar case, the equation of motion of the in plane displacement will be independent of the out of plane displacement. The roughness of the front should be similar to the scalar case, but the out of plane roughness is very different. Following various authors, with the expectation that the local dynamics will be determined by the dominant terms consistent with symmetry, we assume that the crack tip locally prefers mode I loading [15,16]. This implies that the direction of motion of the crack tip can be found by requiring that the curvature at the crack tip, $\kappa$ be proportional to the angular derivative of the $\sigma_{\theta \theta}$ component of the stress tensor, the hoop stress just ahead of the crack tip. As in the scalar case, this just makes sure that the crack is smooth on small 
length scales, and the results on large length scales should be independent of the specific form of this condition. To linear order this leads to the equation for the out of plane displacement

$$
\begin{aligned}
\lambda \kappa & =-\frac{K_{\mathrm{II}}\left(\mathbf{r}_{C F}\right)}{\sqrt{b}}-\chi(x=f(z, t), z) \\
& =-\frac{1}{\sqrt{b}}\left[K_{\mathrm{I}}^{\infty} \partial_{x} h+K_{\mathrm{II}}^{h}\right]-\chi
\end{aligned}
$$

where $K_{\mathrm{II}}^{h}$, which is linear in $h$, is the local mode II loading in the original $x, y, z$ coordinates, obtained from the perturbative solution for the displacement field. Again, $b$ is a microscopic length, $\lambda \sim K_{\mathrm{I}}^{c} \sqrt{b}$, and $\chi$ is a random variable that includes the effects of local variations in the yield stress and the relief of random residual stresses.

Once again $f$ appears only as an implicit variable and $h$ is determined independently of $f$. The Fourier transform of Eq.(15) involves a long range kernel

$$
\tilde{J}_{\mathrm{I}}(q, k)=|q| Y_{\mathrm{I}}(k / q) .
$$

We now see a crucial difference from the scalar case: $\tilde{J}_{\text {I }}$ differs from $\tilde{J}$ by a factor of $1 /|q|^{1 / 2}$, thus the out of plane "stiffness" is much stronger in the mode I case due to the local mode selection. The extra stiffness leads to only logarithmically rough crack surfaces, in striking contrast to what is observed in experiments.

A study of the non-linear terms that we have neglected throughout, leads to the conclusion that they will be irrelevant at long length scales for all the cases considered. In our quasi-static approximation it thus appears that the only way to get rougher surfaces is if the random properties of the solid have long range correlations, which we discuss next.

Residual stresses $\sigma^{r}(\mathbf{r})$, which are present in the material before it cracks, will change the local stress intensity factors at the crack front for a given crack geometry. If $\left\langle\sigma^{r}(\mathbf{r}) \sigma^{r}\left(\mathbf{r}^{\prime}\right)\right\rangle \sim 1 /\left|\mathbf{r}-\mathbf{r}^{\prime}\right|^{\alpha}$ at long distances, dimensional analysis yields additional correlations in the resulting $G^{r}$ and $S_{a}^{r}$, of the form $\Delta(x, z) \sim|z|^{1-\alpha} \hat{\Delta}(|x / z|)$ and $\Upsilon(x, z) \sim|z|^{-\alpha} \hat{\Upsilon}(|x / z|)$. Randomly distributed finite sized defects such as heterogeneities, micro-cracks or loop dislocations each yield stress fields falling off as $1 / r^{3}$ [17] and hence $\alpha=3$. For the roughness of the moving front on scales larger than $\xi$, and the roughness of the crack surfaces in mode $\mathrm{I}$, this is a marginal perturbation which will result in changing the logarithmic roughness to $(\ln )^{2}$. Near the threshold for the crack motion, such correlations are also marginal. But in some disordered materials, growth processes may result in longer range correlations in the residual stresses. As an extreme example, one could consider the stress caused by segments of dislocations placed randomly in the solid with the constraint that they form loops, i.e., that the dislocation density tensor is divergence free. This results in $\alpha=1$ which is a strongly relevant perturbation yielding, in the linear approximation an unphysical roughness exponent of $\zeta_{h}=1$ both for the front and the mode I crack surface. Generally, we have $\zeta_{h}=(3-\alpha) / 2$ for $\alpha<3$, thus very long range correlations with $\alpha \approx 1.4$ would be needed to explain the experiments.

On the basis of our results an explanation of the measured roughness in terms of quasi-static motion of cracks appears unlikely. Only if very long range correlations in residual stresses existed would a quasi-static explanation be viable. However, even if these did occur, one would have replaced the problem of understanding the apparent universality of crack roughness exponents in a wide variety of materials with the problem of why residual stress correlations should be long range and universal. A more appealing alternative is that elastodynamics of the medium plays an essential role. It has been shown that, in the case of a crack front restricted to a plane, the sound waves emitted as it moves changes its behaviour both when it is moving at a finite velocity and near the threshold [12]. Such effects may also play a crucial role in increasing the roughness of the crack surface [18].

We finally note that in thin plates, our quasi-static analysis yields a crack path with a roughness exponent of $\zeta=1 / 2$ for tensile cracks and $\zeta=1$ for tearing cracks; in these situations elastodynamic effects may be less important, but buckling may play a role.

We would like to thank J. R. Rice, E. Bouchaud and J.P. Bouchaud for useful discussions. This work has been supported in part by the NSF via DMR-9106237, 9630064 and Harvard University's MRSEC.

[1] B. B. Mandelbrot, D. E. Possoja, and A. J. Paullay, Nature (London) 308, 721 (1984).

[2] P. Daguier, S. Henaux, E. Bouchaud and F. Creuzet, Phys. Rev. E 53, 5637 (1996) and references therein.

[3] J. J. Mecholsky et al., J. Am. Ceram. Soc. 72, 60 (1989).

[4] J. Schmittbuhl, S. Gentier and S. Roux, Geophys. Lett. 20, 639 (1993).

[5] A. Nakano, R. K. Kalia and P. Vashishta, Phys. Rev. Lett. 73, 2336 (1994); 75, 3138 (1995).

[6] T. Nattermann, S. Stepanow, L.-H. Tang and H. Leschhorn, J. Phys. II (France) 2, 1483 (1992).

[7] O. Narayan and D. S. Fisher, Phys. Rev. B 48, 7030 (1993).

[8] D. Ertaş and M. Kardar, Phys. Rev. E 49, R2532 (1994).

[9] J.-P. Bouchaud, E. Bouchaud, G. Lapasset and J. Planès, Phys. Rev. Lett. 71, 2240 (1993).

[10] J. R. Rice, J. Appl. Mech. 52, 571 (1985).

[11] An account of various proposed criteria is given in H. Bergkvist and L. Guex, Int. J. Fracture 15, 429 (1979).

[12] S. Ramanathan and D. S. Fisher, unpublished.

[13] G. Xu, A. F. Bower and M. Ortiz, Int. J. Solids Structures, 31, 2167 (1994); H. Gao, J. Appl. Mech. 59, 335 (1992).

[14] B. Lawn, Fracture of Brittle Solids, Second Edition, Cambridge University Press, Cambridge 1993.

[15] B. Cotterell and J. R. Rice, Int. J. Fracture 16, 155 (1980).

[16] J. Hodgdon and J. P. Sethna, Phys. Rev. B 47, 4831 (1993).

[17] J. R. Rice, Int. J. Solids Structures 21, 781 (1985).

[18] E. S. C.Ching, J. S. Langer and H. Nakanishi, Phys. Rev. Lett. 76, 1087 (1996), have argued that for certain cohesive laws mode I cracks are unstable on small length scales. 